\title{
Inhibition of AKT signaling uncouples T cell differentiation from expansion for receptor-engineered adoptive immunotherapy
}

Christopher A. Klebanoff, ${ }^{1,2,3}$ Joseph G. Crompton, ${ }^{3,4}$ Anthony J. Leonardi, ${ }^{3}$ Tori N. Yamamoto, ${ }^{3,5}$ Smita S. Chandran, ${ }^{1,2}$ Robert L. Eil, ${ }^{3}$ Madhusudhanan Sukumar, ${ }^{3}$ Suman K. Vodnala, ${ }^{3}$ Jinhui Hu, ${ }^{3,6}$ Yun Ji, ${ }^{3,6}$ David Clever, ${ }^{3}$ Mary A. Black, ${ }^{3}$ Devikala Gurusamy, ${ }^{3}$ Michael J. Kruhlak, ${ }^{7}$ Ping Jin, ${ }^{8}$ David F. Stroncek, ${ }^{8}$ Luca Gattinoni, ${ }^{3,6}$ Steven A. Feldman, ${ }^{3}$ and Nicholas P. Restifo ${ }^{3,9}$

'Center for Cell Engineering and Department of Medicine, Memorial Sloan Kettering Cancer Center (MSKCC), New York, New York, USA. ${ }^{2}$ Parker Institute for Cancer Immunotherapy, New York, New York, USA. ${ }^{3}$ Center for Cancer Research (CCR), National Cancer Institute (NCI), NIH, Bethesda, Maryland, USA. ${ }^{4}$ Department of Surgery, University of California Los Angeles, Los Angeles, California, USA. ${ }^{5}$ mmunology Graduate Group, University of Pennsylvania, Philadelphia, Pennsylvania, USA. ${ }^{6}$ Experimental Transplantation and Immunology Branch and ${ }^{7}$ Experimental Immunology Branch, CCR, $\mathrm{NCI}, \mathrm{NIH}$, Bethesda, Maryland, USA. ${ }^{8}$ Cell Processing Section, Department of Transfusion Medicine, Clinical Center, NIH, Bethesda, Maryland, USA. ${ }^{9}$ Center for Cell-based Therapy, CCR, NCI, NIH, Bethesda, Maryland, USA

Adoptive immunotherapies using T cells genetically redirected with a chimeric antigen receptor (CAR) or T cell receptor (TCR) are entering mainstream clinical practice. Despite encouraging results, some patients do not respond to current therapies. In part, this phenomenon has been associated with infusion of reduced numbers of early memory $T$ cells. Herein, we report that AKT signaling inhibition is compatible with CAR and TCR retroviral transduction of human T cells while promoting a CD62L-expressing central memory phenotype. Critically, this intervention did not compromise cell yield. Mechanistically, disruption of AKT signaling preserved MAPK activation and promoted the intranuclear localization of FOXO1, a transcriptional regulator of T cell memory. Consequently, AKT signaling inhibition synchronized the transcriptional profile for FOX01-dependent target genes across multiple donors. Expression of an AKT-resistant FOX01 mutant phenocopied the influence of AKT signaling inhibition, while addition of AKT signaling inhibition to T cells expressing mutant FOX01 failed to further augment the frequency of CD62L-expressing cells. Finally, treatment of established B cell acute lymphoblastic leukemia was superior using anti-CD19 CAR-modified T cells transduced and expanded in the presence of an AKT inhibitor compared with conventionally grown T cells. Thus, inhibition of signaling along the PIЗK/AKT axis represents a generalizable strategy to generate large numbers of receptor-modified $T$ cells with an early memory phenotype and superior antitumor efficacy.

Authorship note: CAK, JCC, and AJL are co-first authors.

Conflict of interest: The authors have declared that no conflict of interest exists.

Submitted: May 11, 2017 Accepted: October 19, 2017 Published: December 7, 2017

Reference information: JCI Insight. 2017;2(23):e95103. https://doi.org/10.1172/jci. insight.95103.

\section{Introduction}

Adoptive immunotherapy using genetically redirected $\mathrm{T}$ cells expressing a chimeric antigen receptor (CAR) or $\mathrm{T}$ cell receptor (TCR) is poised to enter mainstream clinical practice (1-4). Despite promising results, some patients fail to achieve durable responses using current cell therapies. Multiple factors likely contribute to differences in patient outcomes following adoptive cell transfer (ACT). These include the magnitude of immunosuppression within the tumor microenvironment, tumor intrinsic properties related to antigen or coinhibitory ligand expression, and attributes of the adoptively transferred T cells themselves $(5,6)$. Among these, variables related to the qualities, functions, and numbers of adoptively transferred $\mathrm{T}$ cells are the most amenable to therapeutic manipulation.

Preclinical studies and retrospective analyses of multiple human ACT clinical trials have identified several $\mathrm{T}$ cell intrinsic parameters associated with superior clinical outcomes. For example, the absolute number of infused $\mathrm{T}$ cells correlates with the magnitude of cancer regression in mice (7), a finding corroborated by 
several clinical trials testing tumor infiltrating lymphocytes (TIL) (8-10) and receptor-engineered T cells (11). Similarly, infusion of less-differentiated $\mathrm{T}$ cell subsets that coexpress lymphoid homing markers like CD62L or costimulatory markers such as CD27 are associated with greater tumor regression (12-14). For example, in a recent clinical trial testing the antitumor efficacy of a second-generation anti-CD19 CAR containing a CD28 costimulatory domain, we found that the frequency of CD62L-expressing $\mathrm{T}$ central memory $\left(\mathrm{T}_{\mathrm{CM}}\right)$ cells in the infusion product significantly correlated with the magnitude of $\mathrm{CAR}^{+}$cells in the peripheral blood (15). This value, in turn, directly correlated with the likelihood that a patient would go on to have an objective antitumor response. Thus, the generation of $\mathrm{T}_{\mathrm{CM}}$-phenotype cells remains a major goal of current efforts to improve T cell therapies (16).

Physiologically, the processes of $\mathrm{T}$ cell clonal expansion and cellular differentiation are tightly coupled to one another; as a $\mathrm{T}$ cell population increases in size, a greater proportion of cells become terminally differentiated $(17,18)$. Thus, relatively differentiated $\mathrm{T}$ cell products are generated using many current clinical manufacturing methods for genetically engineered $\mathrm{T}$ cells because these methods focus primarily on the generation of large numbers of antitumor cells $(19,20)$. This is especially true in heavily pretreated patients who have starting apheresis populations containing a paucity of naive/ early memory T cells (21-23).

The coupling of $\mathrm{T}$ cell expansion and differentiation results from the integration of signals arising from multiple sources, including the TCR, costimulatory molecules, and cytokine receptors (24). Together, these signals converge to activate 2 major signal transduction networks within $\mathrm{T}$ cells: the MAPK and PI3K/AKT/mTOR pathways. Genetic and pharmacologic disruption of ERK, a distal component of the MAPK pathway, significantly impairs the proliferation of T cells in both mice (25) and humans (26). By contrast, deletion of Pi3cd (the gene encoding the p1108 catalytic subunit of PI3K enriched in T cells) or inhibition of AKT does not compromise the proliferation or survival of murine $\mathrm{CD}^{+} \mathrm{T}$ cells (27). Consistent with this finding, we recently demonstrated that pharmacologic inhibition of AKT permits the robust expansion of allogenic in vitro-sensitized minor histocompatibility-specific $\mathrm{T}$ cells (28) and melanoma TIL cells (29) with desirable phenotypic and functional attributes. Because genetic engineering using retroviruses requires $\mathrm{T}$ cells to be actively cycling for efficient integration to occur (30), we hypothesized that inhibition of AKT would permit the transduction and expansion of minimally differentiated human $\mathrm{T}$ cells.

Here, using clinical-grade retroviruses for both a CAR and TCR in late-stage clinical development, we show that AKT inhibition using an allosteric kinase inhibitor (AKT Inhibitor VIII; AKTi) (31) is compatible with the activation, expansion, and efficient receptor engineering of human T cells. Mechanistically, the ability of AKTi to allow T cell expansion and transduction while preserving a minimally differentiated CD62L-expressing population was associated with conserved MAPK signaling, the intranuclear accumulation of FOXO1, and the expression of FOXO1-dependent target genes. Even when starting with an unfractionated population of $\mathrm{T}$ cell subsets, AKTi generated receptor-engineered $\mathrm{T}$ cells with desirable genetic and metabolic properties and enhanced in vivo antitumor efficacy relative to conventionally produced $\mathrm{T}$ cells. Thus, inhibition of AKT signaling represents a generalizable strategy to generate large numbers of receptor-modified $\mathrm{T}$ cells with an early memory phenotype, a finding that is now influencing current ACT clinical trials.

\section{Results}

$A K T$ inhibition permits expansion of CD62L-expressing receptor-engineered human $T$ cells. We sought to determine whether pharmacologic inhibition of AKT is compatible with the activation, expansion, and efficient receptor engineering of human $\mathrm{T}$ cells. Therefore, we performed $\mathrm{T}$ cell stimulation and retroviral transduction of a second-generation anti-CD19 CAR (32) in the continuous presence of $1 \mu \mathrm{M}$ of AKTi or a vehicle (Veh) control. To emulate the source of T cells used in the majority of current CD19 CAR clinical trials $(15,33-39)$, we used an unfractionated population of peripheral blood mononuclear cells (PBMC). Both the methods and reagents employed in these experiments were identical to those used for the clinical manufacturing of anti-CD19 CAR-modified T cells $(15,40,41)$ (Figure 1A).

We first confirmed the activity and specificity of the AKTi in peripheral blood $\mathrm{T}$ cells by measuring the phosphorylation state of AKT and ribosomal S6 protein (S6), a downstream mediator of AKT/ mTOR signaling, at rest and serially following $\mathrm{T}$ cell activation (Figure 1, B and C). Within 5 minutes of anti-CD3 exposure, we observed maximal phosphorylation of both the activating $\mathrm{AKT}^{\mathrm{T} 308}$ and $\mathrm{AKT}^{\mathrm{S} 473}$ 
residues and a corresponding increase in phospho-S6 in Veh-treated cells. Additionally, we also observed a dramatic increase in phospho-ERK1/2, a distal component of the MAPK pathway required for T cell proliferation and survival $(25,42)$. In the presence of $\mathrm{AKTi}$, phosphorylation of both $\mathrm{AKT}^{\mathrm{T} 308}$ and $\mathrm{AKT}^{\mathrm{S} 473}$ were significantly suppressed to levels comparable with that of unstimulated T cells. Phospho-S6 was partially but not completely attenuated, a finding reflecting the multiple kinases that may activate the mTOR/S6 pathway independently of AKT (43). By contrast, induction of phospho-ERK1/2 was entirely unperturbed in AKTi-treated T cells.

In keeping with the preservation of ERK signaling, we measured no differences in either the growth rate kinetics or overall expansion of anti-CD19 CAR-modified cells grown in the presence of AKTi compared with Veh control (Supplemental Figure 1; supplemental material available online with this article; https://doi.org/10.1172/jci.insight.95103DS1 and Figure 1D). Because $\gamma$-retroviruses require active cell division for efficient gene integration (30), we next tested whether AKTi impacted the transduction efficiency of peripheral blood T cells. Critically, we found that AKTi did not significantly alter the ability to introduce the anti-CD19 CAR into either $\mathrm{CD}^{+}$or $\mathrm{CD}^{+} \mathrm{T}$ cells (Figure $1 \mathrm{E}$ ). Consistent with comparable transduction efficiencies, we also found that AKTi exposure during anti-CD19 CAR manufacturing did not impair antigen-specific effector functions once the drug was removed from the culture media. For example, the magnitude of IFN $\gamma$ release following coculture with CD19+ tumor cells (Supplemental Figure 2), as well as cytolytic capacity (Supplemental Figure 3), was similar with both AKTi- and Veh-expanded T cells. By contrast, unstimulated CAR T cells or T cells cocultured with CD19- tumor cells produced minimal to no IFN $\gamma$ and did not mediate tumor lysis, regardless of initial culture conditions.

Activated T cells lose expression of CD62L, a lymphoid homing molecule and key marker of highly potent $\mathrm{T}$ cell subsets for adoptive immunotherapy $(5,15,44)$, in an AKT1/2-dependent manner (45). We therefore evaluated whether inhibition of AKT could preserve expression of CD62L on CAR-engineered $\mathrm{T}$ cells. We found that AKTi significantly preserved CD62L expression on CAR-modified $\mathrm{T}$ cells compared with cells expanded in Veh control (Figure 1, F and G). Some CAR constructs containing a CD28 costimulatory domain have recently been shown to exhibit persistent AKT signaling in the absence of cognate antigen through autosignaling $(46,47)$. We therefore also tested whether AKTi could benefit gene engineering with a TCR, an antigen receptor not known to autosignal. We found that AKTi maintained CD62L expression without compromising transduction efficiency or $\mathrm{T}$ cell expansion using a TCR specific for the cancer-germline antigen NY-ESO-1 (48-50) (Supplemental Figure 4).

Finally, we tested whether AKTi was compatible with the use of anti-CD3/CD28 microbead stimulation of isolated memory $\mathrm{T}$ cell subsets, a manufacturing practice done by some academic and commercial cell therapy groups (51). Similar to results using an unfractionated PBMC population activated with OKT3, we found that AKTi did not interfere with microbead-driven $\mathrm{T}$ cell expansion but, nonetheless, could significantly preserve CD62L expression on isolated $\mathrm{T}_{\mathrm{CM}}$ cells (Supplemental Figure 5). By contrast, expansion of effector memory $\mathrm{T}$ cells $\left(\mathrm{T}_{\mathrm{EM}}\right)$ in AKTi failed to increase the frequency of $\mathrm{T}_{\mathrm{CM}}$ cells, an expected finding given the lineage relationship between these subsets (52). Taken together, we conclude that pharmacologic disruption of AKT signaling permits the activation, expansion, and efficient retroviral transduction of human peripheral blood T cells with either a CAR or TCR, while simultaneously promoting the expression of CD62L.

AKT signaling blockade limits $T$ cell differentiation and promotes intranuclear FOXO1. We next sought to obtain a more global assessment of the influence of AKTi on the resulting population of modified $\mathrm{T}$ cells. We therefore performed a transcriptomic analysis of unfractionated peripheral blood $\mathrm{T}$ cells from 3 separate donors at rest and following 10 days of expansion and retroviral transduction with an anti-CD19 CAR in the presence of AKTi or Veh control. Despite the heterogenous distribution of T cell subsets present in the starting populations (data not shown), unsupervised hierarchical clustering revealed the dominant influence of AKTi exposure on the resulting pattern of gene expression. Inhibitor-treated cells clustered together in all donors following activation, expansion, and genetic modification (Figure 2A). In total, we identified 4,960 and 4,732 significantly up- and downregulated transcripts, respectively, in CAR-modified T cells expanded in AKTi compared with Veh control $(P<0.01$, FDR $<$ $5 \%$; Supplemental Table 1 and 2). This indicated that the influence of the drug had a balanced effect on repressing and activating gene expression in peripheral blood $\mathrm{T}$ cells. 
A

PBMC

$n=3-6$ donors
Time (d)

0 (d)

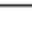

$+2$

\begin{tabular}{|c|c|}
\multicolumn{1}{c|}{} & RV Td \\
\hline Anti-CD3 (50ng mL-1) & \\
\hline \hline IL-2 (300 IU mL-1) \\
\hline \hline Vehicle \\
\hline AKTi $(1 \mu \mathrm{M})$ \\
\hline
\end{tabular}

Kinase phosphorylation

Enumeration

$R V$ Td efficiency

Co-culture

Surface phenotype

Gene expression

Metabolomics

Tumor treatment
B

$=$ pre-stimulation $\square=$ post-stimulation, Veh

AKT/mTOR signaling

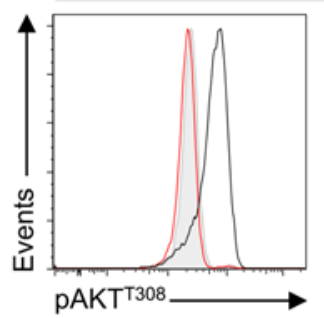

C Veh $\mathrm{AKTi}$

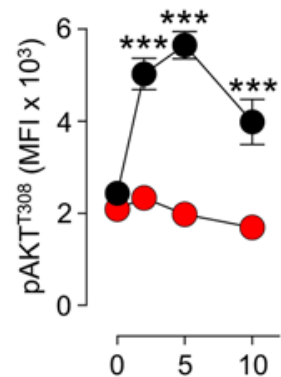

Time after anti-CD3 (min)
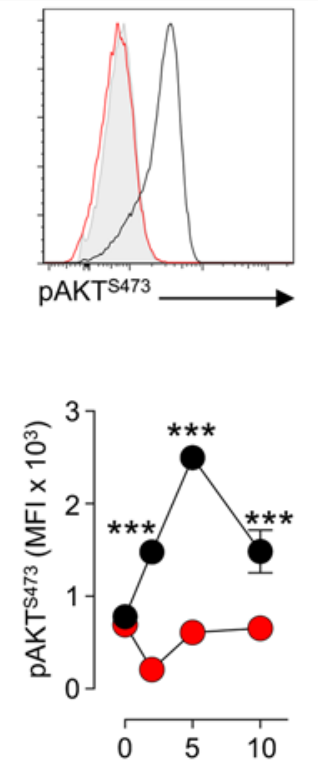

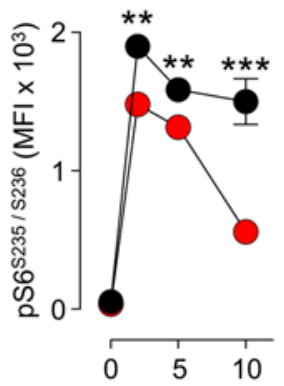

$\mathbf{F}$
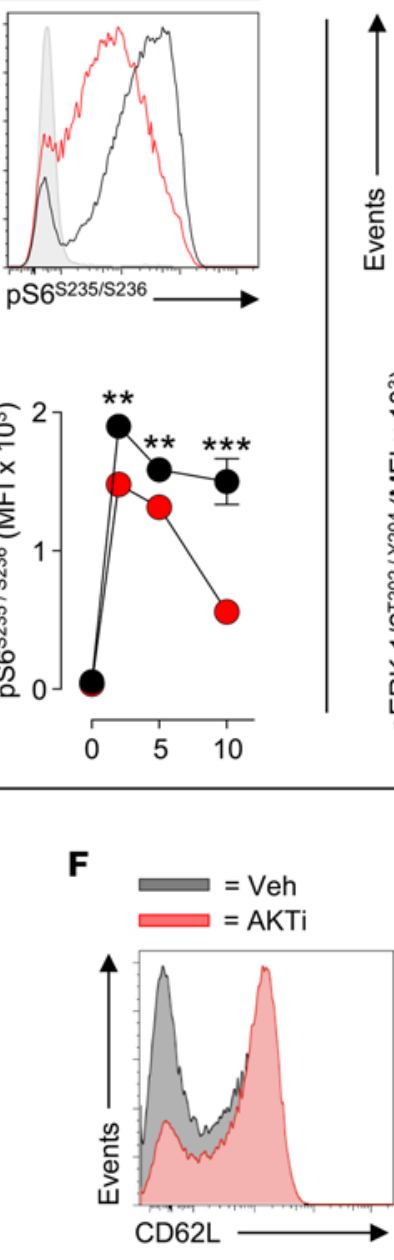

= post-stimulation, $\mathrm{AKTi}$

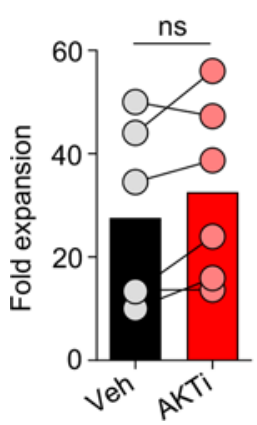

E

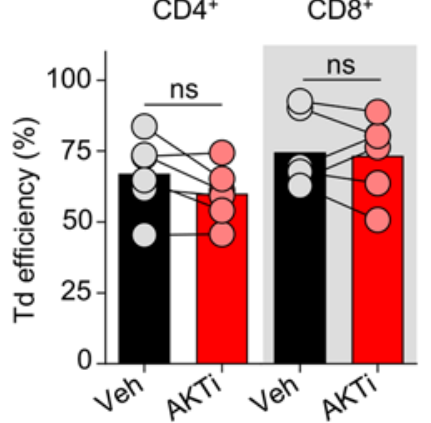

G

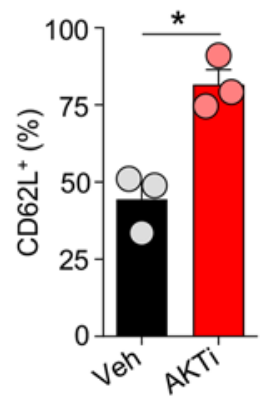

Figure 1. Pharmacologic inhibition of AKT signaling permits expansion of CD62L-expressing receptor-engineered human peripheral blood T cells. (A) Schema for the anti-CD3 (50 $\mathrm{n} \mathrm{ml}^{-1}$ ) activation, retroviral transduction (RV Td), and expansion of human peripheral blood T lymphocytes (PBL) in the continuous presence of IL-2 (300 IU ml-1) and AKT inhibitor VIII (AKTi; $1 \mu \mathrm{M}$ ) or vehicle control (Veh). (B) Representative phosphoflow cytometry plots and (C) graphical summary of the time-dependent phosphorylation of kinases involved AKT/mTOR or MAPK signaling in PBL expanded in the presence or absence of AKTi immediately prior to and following stimulation with an anti-CD3 antibody. Results from 1 of 2 representative experiments are displayed. (D) Fold expansion and (E) transduction efficiency of unfractionated PBL genetically engineered with a second-generation $28 z$ anti-CD19 chimeric antigen receptor (CAR) following ex vivo expansion over 10d in the continuous presence IL-2 and AKTi or Veh. Pooled results from 6 independent donors are shown after gating on viable, transduced CD3 ${ }^{+}$CD4 ${ }^{+}$and $\mathrm{CD3}^{+} \mathrm{CD}^{+}$cells. (F) Representative FACS plot and (G) graphical summary of CD62L expression on CAR-modified PBL expanded for 10d in AKTi or Veh control. Results shown in panels $\mathbf{D}-\mathbf{G}$ are based on patient-derived samples, while results in panels $\mathbf{B}$ and $\mathbf{C}$ used healthy donor (HD) T cells. Data in panels $\mathbf{C}-\mathbf{E}$ and $\mathbf{G}$ are presented as mean \pm SEM with $n=3, n=6, n=6$, and $n=3$ per condition, respectively. All statistical comparisons were performed using an unpaired 2-tailed Student's $t$ test. ${ }^{* *} P<0.001 ;{ }^{* *} P<0.01 ;{ }^{*} P<0.05$. 
Examination of specific transcripts revealed that $S E L L$, the gene encoding CD62L, was among the top $2 \%$ most enriched genes following AKTi exposure in all donors (Figure $2 \mathrm{~B}$ ). This finding was consistent with protein expression data we measured for CD62L using FACS analysis (Figure 1F and Supplemental Figure 4D). We further found that other established targets of AKT signaling associated with $\mathrm{T}$ cell trafficking and persistence, including $\operatorname{IL} 7 R(27,53,54), K L F 2$ (27), and $S 1 P R 1$ (27), were systematically overexpressed in AKTi-expanded T cells. Expression of multiple costimulatory members of the TNF receptor superfamily linked to enhanced CAR T cell function, such as CD28 and ICOS (46), were also differentially retained by AKTi-treated cells. Conversely, we found that genes associated with acquisition of $\mathrm{T}$ cell effector functions, including the cytotoxic proteases GZMA, GZMB, GZMH, and GZMM and the death/differentiation-inducing ligand FASLG $(21,55,56)$, were suppressed by AKT inhibition. Moreover, genes involved in apoptosis induction, such as $B A D, B A X$, and $T R A I L$, were also downregulated. Functionally, reduction in this latter group of genes was associated with a reduced propensity of inhibitor-treated $\mathrm{T}$ cells to undergo apoptosis following expansion compared with cells grown in control media (Supplemental Figure 6). Thus, we found that AKT signaling blockade had a broad influence on the expression of multiple genes associated with $\mathrm{T}$ cell differentiation and survival.

Patients with previously treated B cell malignancies have significant skewing of their T cell compartment toward highly differentiated subsets with compromised therapeutic potential (21-23), a finding likely reflecting the influence of prior lymphodepleting chemotherapy. This situation can be further exacerbated by conventional ex vivo cell manufacturing processes $(19,20,57,58)$. We therefore next asked whether AKTi preserves key markers of cellular differentiation and effector function relative to an unstimulated starting population of $\mathrm{T}$ cells. We compared the magnitude of expression for $I L 7 R, K L F 2, C D 28$, and GZMB in T cells from the same donor following collection from the peripheral blood and after expansion and genetic engineering in AKTi or Veh control. As expected (59), we found that $\mathrm{T}$ cell activation under standard conditions resulted in the acquisition of the effector molecule GZMB and a significant loss of the naive $\mathrm{T}$ cell/memory $\mathrm{T}$ cell-associated markers $I L 7 R, K L F 2$, and $C D 28$ (Figure 2C and Supplemental Figure 7). By contrast, expansion in AKTi prevented the acquisition of $G Z M B$, while minimizing the loss of $I L 7 R$ and preserving the expression of $K L F 2$ and $C D 28$ to levels comparable with unstimulated T cells.

We noted that several of the differentially expressed genes under AKTi treatment, including $I L 7 R$, $K L F 2, S 1 P R 1$, and $S 1 P R 2$, are directly regulated by the transcription factor FOXO1 (60). In mice, FOXO1 has recently been identified as a key transcriptional regulator required for $\mathrm{CD} 8^{+} \mathrm{T}$ cell memory formation and persistence (61-63). Across species, FOXO1 transcriptional activity is controlled through posttranslational modifications, most notably phosphorylation of a series of 3 conserved serine and threonine residues (64). In human cancer cell lines, AKT is capable of phosphorylating FOXO1 at these sites, causing cytosolic sequestration and preventing DNA binding (65). To test whether AKT inhibition results in the nuclear accumulation of FOXO1 in activated human T cells, we performed confocal immunofluorescence imaging of this protein in T cells stimulated for 4 days in the presence of titrated concentrations of AKTi or a Veh control. Using costaining of Hoechst and phalloidin to respectively define the boundaries of the nucleus and cytoplasm, we found that exposure to AKTi resulted in a significant, dose-dependent intranuclear localization of this transcription factor (Figure 2, D and E). The differential subcellular localization of FOXO1 in activated T cells expanded in the presence or absence of AKTi was further confirmed by Western blot analysis of protein fractions isolated from the nucleus versus the cytoplasm (Supplemental Figure 8). The accumulation of FOXO1 was not attributable to differences in FOXO1 gene expression, as similar quantities were produced by AKTi- and Veh-treated T cells (Figure 2F). Collectively, these data indicated that AKTi caused a consistent and pervasive change in gene expression in CD19 CAR-engineered T cells, resulting in a population with desirable phenotypic, functional, and cell-survival attributes. These alterations were, in turn, associated with the nuclear localization of FOXO1, a key transcription factor associated with $\mathrm{T}$ cell memory formation.

Constitutively active FOXO1 augments CD62L expression independently of AKTi. Given the association between AKTi exposure and the subcellular localization of FOXO1, we next sought to understand whether active FOXO1 was sufficient to maintain CD62L expression on gene-engineered T cells. The similar levels of FOXO1 gene expression measured in AKTi- and Veh-treated cells suggested that a posttranslational mechanism might control FOXO1 activity in human T cells. We therefore created a mutant variant of FOXO1 (hereafter termed FOXO1 ${ }^{\mathrm{AAA}}$ ) in which target sites of AKT phosphorylation — including the T24, S256, and S319 residues — were each substituted for a phosphorylation-resistant 
A

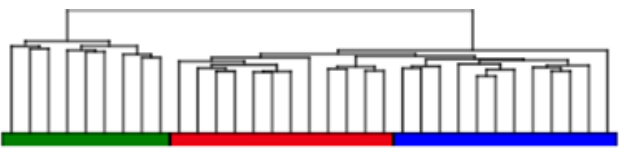
baseline

$\mathrm{AKTi}$

Veh
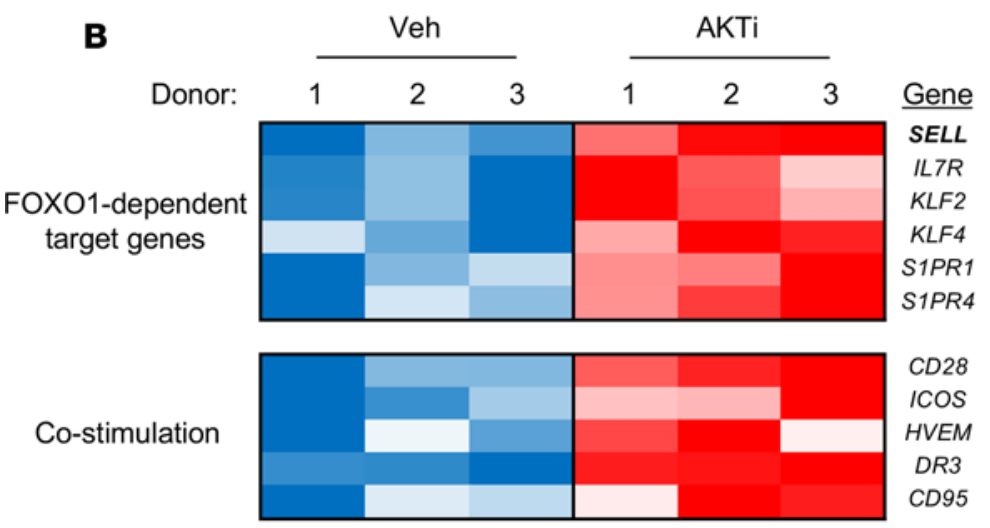

CD28

ICOS

HVEM

$D R 3$

CD95

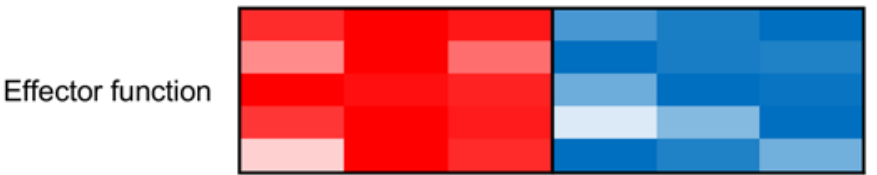

GZMA

GZMB

$G Z M H$

GZMM

FASLG

Apoptosis

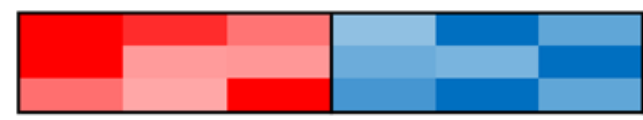

$B A D$

$B A X$

TRAIL

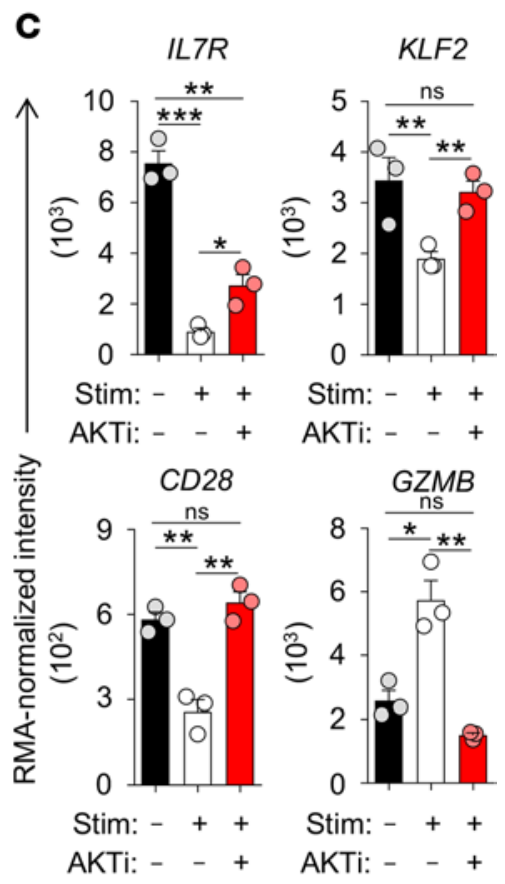

low

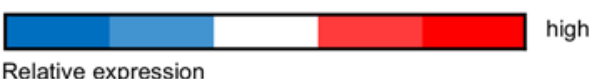

AKTi: - - +

Relative expression

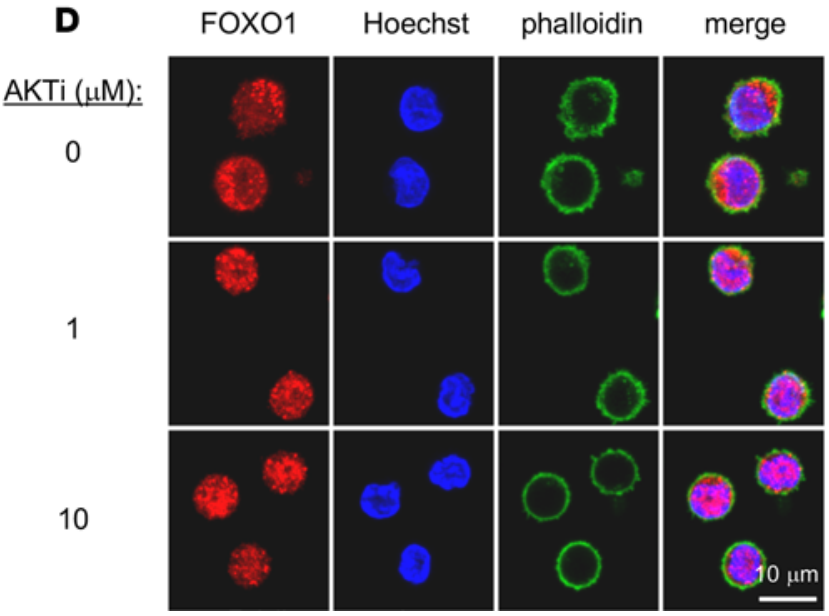

E

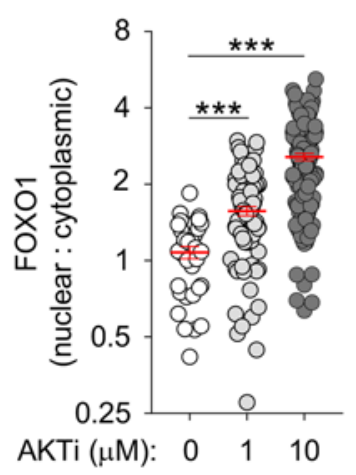

$\mathbf{F}$

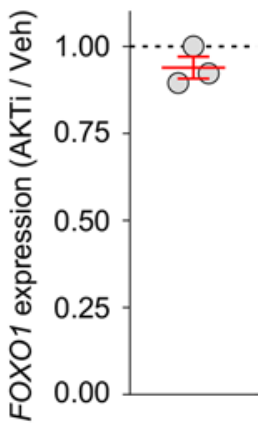

Figure 2. Blockade of AKT signaling minimizes gene expression changes associated with human T cell differentiation and promotes nuclear accumulation of the transcription factor FOX01. (A) Unsupervised hierarchical clustering of gene expression from peripheral blood T cells at baseline and 10d after stimulation, retroviral transduction (RV Td) with an anti-CD19 chimeric antigen receptor (CAR), and expansion in the continuous presence of IL-2 (300 IU ml-1) and AKT inhibitor VIII (AKTi; $1 \mu \mathrm{M})$ or a vehicle control (Veh). Clustering was based on all significantly expressed genes (1-way ANOVA, FDR-corrected $P<0.05$ ) using a Pearson correlation. T cells were derived from 3 independent donors using an $n=3-4$ replicates per donor, per time point, and per condition. (B) Heat map of expression for selected genes that are direct targets of the transcription factor F0X01 or genes involved in T cell costimulation, effector function, and apoptosis. Red and blue colors indicate relative increased and decreased expression, respectively. Each column represents the average expression of a gene from an individual donor, and each row represents the indicated gene. (C) Robust multichip analysis-normalized (RMA-normalized) intensity of selected FOX01-target genes and genes associated with T cell differentiation at baseline and 10d after ex vivo expansion in the continuous presence of AKTi or Veh. The bar graphs represent the mean \pm SEM of $n=3$ overlaid biological replicates per condition from a single donor and are representative of experiments observed with all 3 independently analyzed donors. (D) Representative confocal immunofluorescence images and (E) summary graph quantifying the dose-dependent intranuclear accumulation of FOX01 $4 \mathrm{~d}$ following T cell activation and expansion in indicated concentrations of AKTi or Veh control. Red, FOX01; blue, Hoechst; and green, phalloidin. Scale bar: $10 \mu \mathrm{m}$. Data shown are representative of 2 independently performed experiments using 2 separate donors. (F) The ratio of gene expression 
levels for FOXO1 in AKTi- versus Veh-treated cells from $n=3$ independently evaluated donors. Dashed horizontal line indicates an expression ratio of 1. Data shown in panels $\mathbf{A}-\mathbf{C}$ and $\mathbf{F}$ are from patient-derived T cells, and panels $\mathbf{D}$ and $\mathbf{E}$ are from healthy donors (HDs). Statistical comparisons in panels $\mathbf{C}$ and $\mathbf{E}$ were performed using an unpaired 2-tailed Student's $t$ test corrected for multiple comparisons by a Bonferroni adjustment. ${ }^{* * *} P<$ $0.001 ;{ }^{*} P<0.01 ;{ }^{*} P<0.0167$.

alanine (Figure 3A). This approach was shown previously to enforce nuclear accumulation of the protein $(66,67)$. To permit the tracking of $\mathrm{T}$ cells modified with FOXO1 ${ }^{\mathrm{AAA}}$, we cloned the mutant gene into a retroviral vector containing a Thy1.1 reporter sequence (Figure 3B). We then compared by FACS the expression of CD62L and other T cell surface markers associated with $\mathrm{T}$ cell memory or exhaustion in Thy $1.1^{+}$transduced cells grown with or without AKTi. An aliquot of T cells from the same donor contemporaneously transduced with an empty vector containing only Thy 1.1 were used as negative controls. In repeated experiments, we found that the Thy $1.1^{+}$population of $\mathrm{T}$ cells transduced with FOXO1 ${ }^{\mathrm{AAA}}$ differentially expressed CD62L compared with the Thy $1.1^{-}$population (Supplemental Figure 9). This indicated both that the AKT-resistant FOXO1 ${ }^{\mathrm{AAA}}$ variant was sufficient to induce $\mathrm{CD} 62 \mathrm{~L}$ expression on engineered $\mathrm{T}$ cells and that its influence occurred in a cell-intrinsic manner. We next tested whether constitutively active FOXO1 was sufficient to maintain CD62L expression on T cells independent of AKT activity. In the same experiment, we compared the expression of CD62L on Thy $1.1^{+} \mathrm{T}$ cells transduced either with FOXO1 ${ }^{\mathrm{AAA}}$ or the empty vector control expanded in the presence or absence of AKTi. Consistent with our previous results, T cells transduced with the empty vector and grown in AKTi resulted in a greater frequency of $\mathrm{CD} 62 \mathrm{~L}^{+}$cells compared with cells grown in Veh control (Figure 3C). Expression of FOXO ${ }^{\mathrm{AAA}}$ in $\mathrm{T}$ cells expanded in control media generated a similar frequency of $\mathrm{CD} 62 \mathrm{~L}^{+} \mathrm{T}_{\mathrm{CM}}$-like cells as $\mathrm{T}$ cells transduced with the empty vector control and expanded in AKTi. However, the addition of AKTi to FOXOAAA-transduced $\mathrm{T}$ cells failed to further increase the $\mathrm{CD}^{2} 2 \mathrm{~L}^{+}$population (Figure 3D). These findings were not restricted solely to $\mathrm{CD} 62 \mathrm{~L}$, as expression of $\mathrm{CD} 28$, another memory-associated marker, followed a similar pattern. Conversely, either AKTi alone or $\mathrm{T}$ cells transduced with FOXO1 ${ }^{\mathrm{AAA}}$ in control media resulted in a comparable downregulation of the coinhibitory marker TIM3 relative to Veh-expanded cells. Taken together, these data indicated that the ability of AKTi to generate a population of human $C D 62 \mathrm{~L}^{+} \mathrm{T}_{\mathrm{CM}}$-like cells occurs through a FOXO1-dependent mechanism.

AKTi limits glycolytic metabolism in human T cells transduced with an anti-CD19 CAR. Recently, several groups have reported that the metabolic fitness of anti-CD19 CAR-engineered $\mathrm{T}$ cells correlates with antitumor efficacy $(58,68,69)$. Because AKT plays a key role in modulating nutrient uptake and cellular metabolism across a range of cell types (70), we next asked whether AKTi alters the metabolic profile of anti-CD19 CAR-modified cells. First, we performed a liquid chromatography-tandem mass spectrometry-based (LC-MS/MS-based) metabolomic analysis to obtain a global snapshot of the relative intracellular concentrations of metabolites in anti-CD19 CAR-modified cells expanded in AKTi or Veh control. In total, we detected 308 distinct metabolites using this assay. Consistent with the well-characterized function of AKT in metabolic regulation, principal component analysis (PCA) revealed that the metabolite profiles of $\mathrm{T}$ cells clustered based on whether they were expanded in the presence or absence of AKTi (Figure 4A). This finding suggested that AKT inhibition resulted in a systematic perturbation of human $\mathrm{T}$ cell metabolism, regardless of the original patient source. Examination of specific intracellular metabolites detected in AKTi- versus Veh-treated cells demonstrated that lactate, the acidic end product of aerobic glycolysis, was among the most significantly downregulated metabolites in the presence of AKTi (average fold change $[\mathrm{FC}]=0.62, P<1 \times 10^{-7}$ ) (Figure $4 \mathrm{~B}$ and Supplemental Table 3). Consistent with this finding, we noted that AKT inhibition systematically suppressed color changes associated with acidification in phenol red-containing culture media (Supplemental Figure 10). These observations prompted us to next ask whether AKTi might suppress aerobic glycolysis in human $\mathrm{T}$ cells.

To functionally test whether AKT inhibition altered glycolysis in human peripheral blood lymphocytes, we performed 2 complementary assays. First, we measured the accumulation of 2-(N-[7-nitrobenz2-oxa-1,3-diazol-4-yl]amino)-2-deoxyglucose (2-NBDG) in T cells activated and expanded in the presence or absence of AKTi; 2-NBDG is a fluorescent glucose analog that permits the quantification of glucose incorporation into living cells (71), including human T cells (72). We found that AKTi significantly reduced 2-NBDG fluorescence intensity (Figure 4C), suggesting that AKT inhibition limited the uptake 
A $\quad$ AKT S/T

$\begin{array}{llll}\text { phosphorylation sites: } & \text { T24 } & \text { S256 } & \text { S319 }\end{array}$

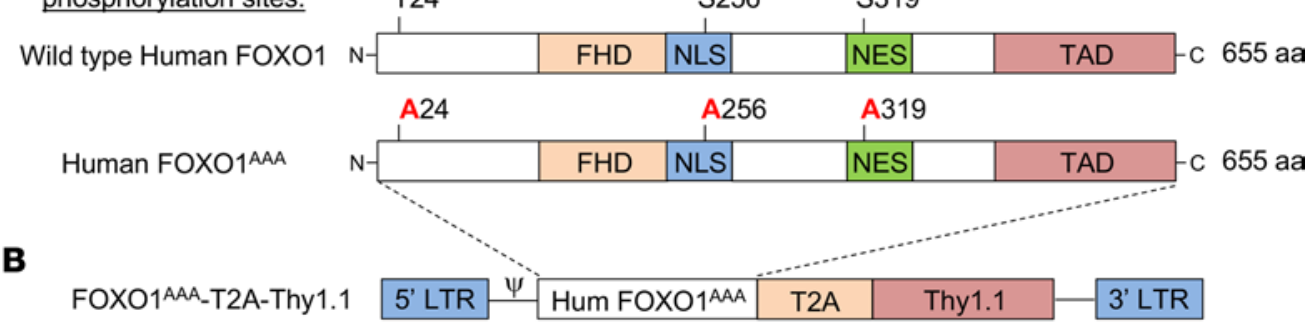

or

Empty vector Thy1.1 5' LTR ${ }^{\psi}$ Thy1.1

C

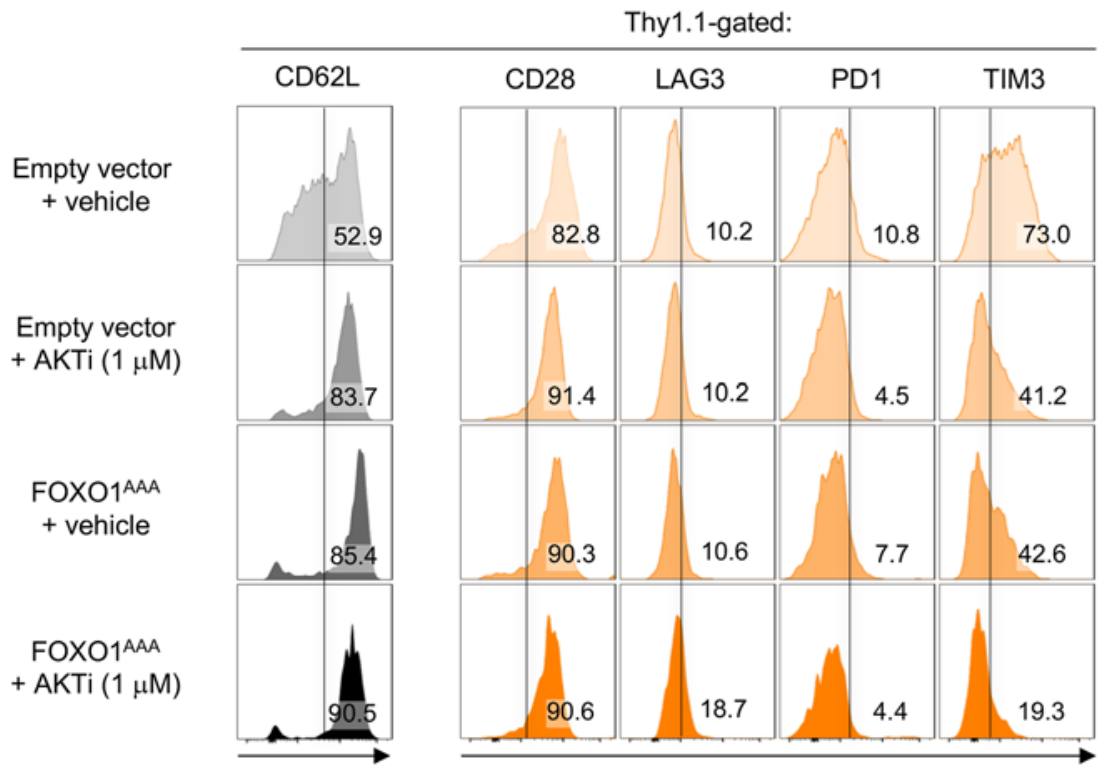

D
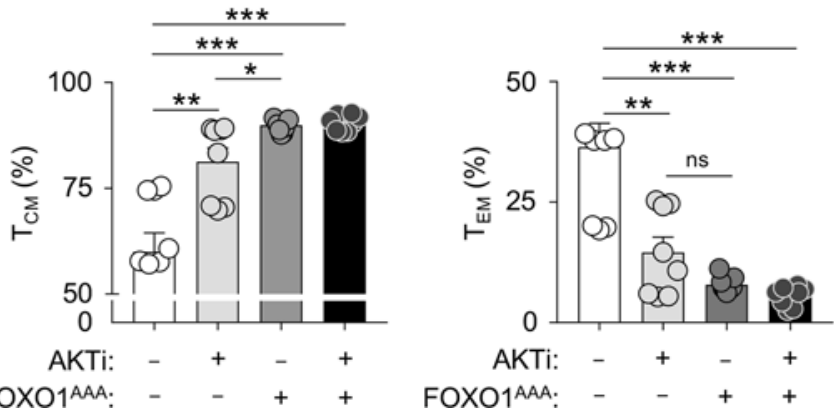

Figure 3. Expression of a constitutively active form of FOX01 augments CD62L expression independently of AKTi. (A) Schematic diagram of WT human FOXO1 protein highlighting 3 serine/threonine residues that serve as AKT phosphorylation sites and an alanine substituted variant (FOXO1 ${ }^{A A}$ ) refractory to AKT phosphorylation. Shaded boxes represent specific functional domains within the FOX01 protein. FHD, forkhead domain; NLS, nuclear localization signal; NES, nuclear export signal; TAD, transcriptional activation domain. (B) Schematic illustration of the MSCV retroviral constructs containing FOX01 $\mathrm{AAA}$ and a Thy1.1 reporter separated by a T2A ribosomal skip sequence (FOX01 ${ }^{\mathrm{AA}}$-T2A-Thy1.1) or an empty vector Thy1.1 (Empty-Thy1.1) control. (C) Expression of CD62L, CD28, LAG3, PD1, and TIM3 on Thy1.1-gated T cells $5 \mathrm{~d}$ after stimulation and retroviral transduction with FOXO1 ${ }^{\mathrm{AAA}}$ T2A-Thy1.1 or Empty-Thy1.1 control in the presence or absence of AKTi $(1 \mu \mathrm{M})$ and IL-2 (300 IU ml-1). Results from 1 of 2 representative experiments are shown. (D) Summary bar graphs and overlaid individual data points showing the distribution of $T_{C M}$ and $T_{E M}$ subsets in $T$ cells expanded in the presence or absence of AKTi following transduction with FOXO1AAA-T2A-Thy1.1 or Empty-Thy1.1. Each bar graph represents the mean \pm SEM of independent replicates from $n=8$ independently performed experiments using healthy donor (HD) T cells. Statistical comparisons performed using an unpaired 2-tailed Student's $t$ test corrected for multiple comparisons by a Bonferroni adjustment. ${ }^{* *} P<0.001 ;{ }^{*} P<0.01 ;{ }^{*} P<0.0167$. 
A

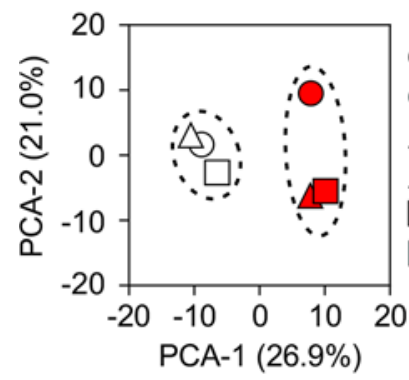

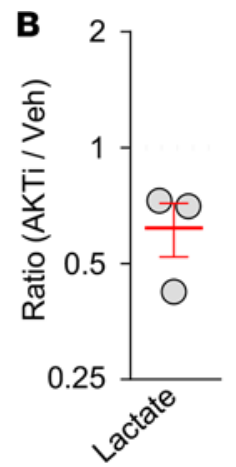

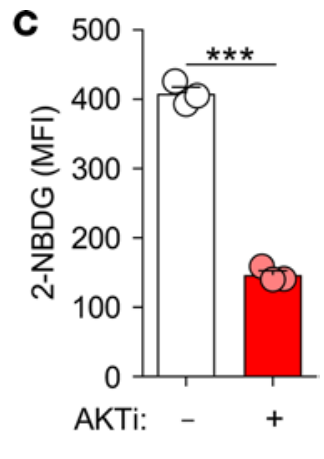

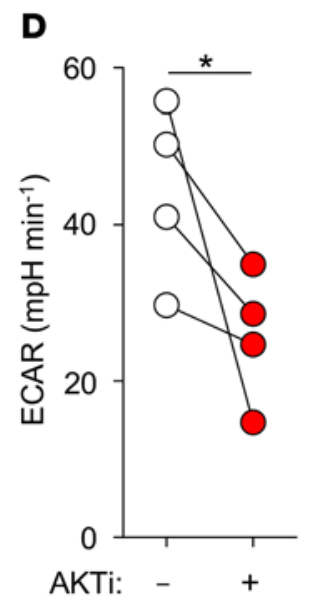

E

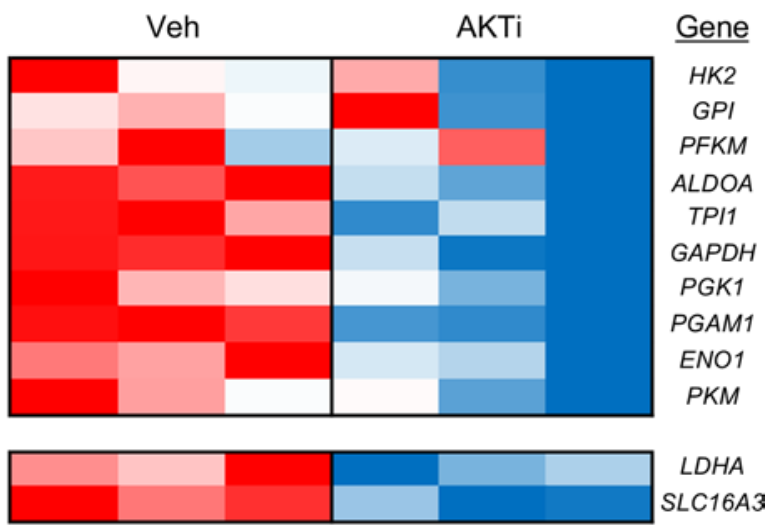

low

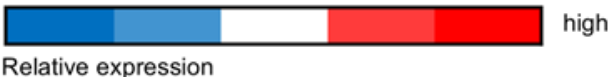

Relative expression

Figure 4. AKTi limits the acquisition of a glycolytic metabolism in human peripheral blood T cells transduced with an anti-CD19 CAR. (A) Two-dimensional principal component analysis (PCA) visualizing the variation in 308 metabolites detected using a metabolomic assay in anti-CD19 chimeric antigen receptor (CAR) transduced T cells expanded in AKTi or vehicle control (Veh). Percentages indicate the amount of variance captured within each principal component. Results are shown from 3 independently evaluated donors using the average of $n=5$ replicates per condition and donor. (B) Ratio of lactate, an end glycolytic intermediate, detected in anti-CD19 CAR transduced T cells expanded in the continuous presence of AKTi versus Veh. The mean ratio \pm SEM from $n=3$ independently evaluated donors is shown with the dashed line indicating a lactate ratio of 1. (C) 2-(N-[7-nitrobenz-2-oxa-1,3-diazol-4-yl]amino)-2-deoxyglucose (2-NBDG) uptake and (D) extracellular acidification rate (ECAR) 5d after stimulation and expansion of peripheral blood T cells in the continuous presence of AKTi or Veh control. Results shown in panel $\mathbf{C}$ are representative of 3 independently performed experiments; results in panel $\mathbf{D}$ are based on cultures from $n=4$ independently evaluated donors. (E) Heat map of gene expression for the 10 enzymes involved in glycolysis and the 2 primary lactate exporters, LDHA and SLC16A3, found in lymphocytes. Each column represents the average expression of a gene from 3 independently evaluated patient donors, and each row represents the indicated gene. Red and blue colors indicate relative increased and decreased expression, respectively. Data shown in panels $\mathbf{A}, \mathbf{B}$, and $\mathbf{E}$ are from patient-derived $\mathrm{T}$ cells, and panels $\mathbf{C}$ and $\mathbf{D}$ are from healthy donors (HDs). Statistical comparisons in panels $\mathbf{C}$ and $\mathbf{D}$ were performed using an unpaired 2-tailed Student's $t$ test. ${ }^{* *} P<0.001 ;{ }^{*} P<0.05$.

of glucose into activated $\mathrm{T}$ cells. Next, we recorded the extracellular acidification rate (ECAR) of $\mathrm{T}$ cells that had been grown with or without AKTi. ECAR measures the extrusion of hydrogen ions by T cells into media as a function of time and, therefore, can be used as an indirect measure of glycolytic flux and lactate production by lymphocytes. Consistent with results from our metabolomic assay demonstrating reduced lactate within AKTi-treated cells, we found that AKT inhibition significantly reduced ECAR across 4 independently evaluated donors (Figure 4D).

AKT can regulate glycolysis both directly through posttranslational modification and indirectly through regulation of gene expression. For example, AKT activity can promote the trafficking and surface expression of GLUT1, the primary glucose transporter in T cells (73). Further, AKT can also enhance the activity of glycolytic enzymes, such as hexokinase II (74). To test whether AKTi might also suppress glycolysis indirectly through changes in gene expression, we measured the transcript levels for all 10 enzymes involved in glycolysis in anti-CD19 CAR-modified T cells expanded for 10 days in AKTi or Veh control. We found that inhibition of AKT systematically suppressed most genes encoding glycolytic enzymes (Figure 4E). Additionally, we also found that genes involved in lactate metabolism 
A

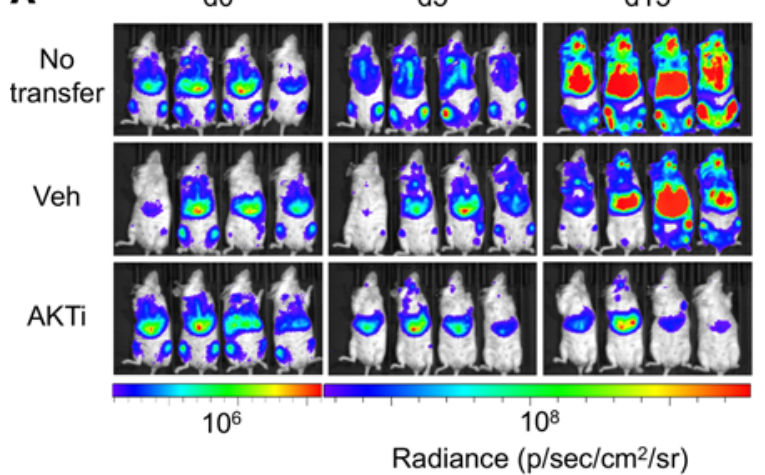

Figure 5. Anti-CD19 CAR-modified T cells expanded in the presence of AKTi treat established leukemia superiorly to conventionally grown T cells. (A) In vivo bioluminescent imaging and (B) overall survival of NOD scid $\gamma$ (NSG) immunodeficient mice bearing established, systemic, NALM6-GL leukemia xenografts following adoptive transfer of anti-CD19 chimeric antigen receptor-modified (anti-CD19 CAR-modified) peripheral blood T cells expanded in the presence of AKTi $(1 \mu \mathrm{M})$ or vehicle (Veh) control. Data shown in panel $\mathbf{A}$ is from $\mathrm{T}$ cells using a patient donor that is representative of findings in all 3 patient donors; data shown in panel $\mathbf{B}$ are the pooled survival results from $n=4-5$ mice per condition per donor using cells derived from 3 patient donors. Differences in overall survival were determined using the Mantel-Cox test. ${ }^{* *} P<0.001 ;{ }^{*} P<0.05$.

B

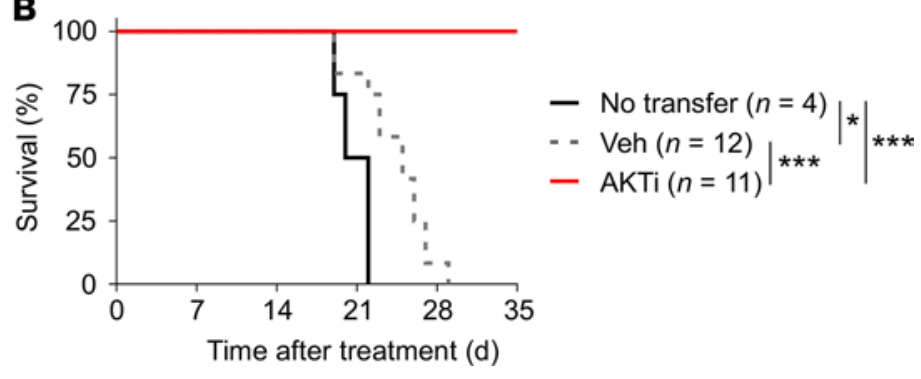

and export - including $L D H A$, the gene encoding lactate dehydrogenase $\mathrm{A}$, and the lactate transporter SLC16A3 (also known as MCT4) - were suppressed in AKTi- versus Veh-treated cells (Figure 4E). By contrast, expression of $C P T 1 A$, a key enzyme involved in fatty acid oxidation in T cells, was not significantly altered by AKTi (Supplemental Figure 11). We therefore conclude that AKTi represses glycolysis in human anti-CD19 CAR-engineered T cells both functionally and through perturbations in the gene expression of key metabolic enzymes.

Anti-CD19 CAR-engineered T cells expanded in AKTi treat leukemia superiorly. The phenotypic, metabolic, and gene expression profiles we measured in anti-CD19 CAR-engineered T cells expanded with AKTi were all consistent with $\mathrm{T}$ cells predicted to have superior antitumor capacities. Therefore, we next asked whether adoptive transfer of anti-CD19 CAR-engineered human T cells grown with AKTi would result in superior in vivo antitumor efficacy. We generated anti-CD19 CAR-modified T cells in the presence of AKTi or Veh control using T cells derived from the PBMC of 3 independently evaluated donors using the clinical cell manufacturing protocol described in Figure 1A. Immunodeficient NOD scid $\gamma$ (NSG) mice with established and widely disseminated NALM6 acute lymphoblastic leukemias (ALL) expressing a luciferase reporter gene were treated with $1 \times 10^{6}$ CAR-modified T cells from a single donor or were left untreated as controls. We did not administer exogenous IL-2 to support the CAR-modified $\mathrm{T}$ cells after infusion to emulate the conduct of current clinical trials. Consistent with the clinical efficacy of anti-CD19 CAR-modified T cells for patients with ALL observed across clinical trials $(34-36,51)$, we found that $\mathrm{T}$ cells expanded under conventional conditions caused a substantial delay in tumor growth compared with animals that did not receive cell transfer (Figure 5A). Across all 3 donors tested, however, the activation, retroviral transduction, and expansion of anti-CD19 CARengineered $\mathrm{T}$ cells in the presence of AKTi resulted in a significant improvement in animal survival compared with animals receiving Veh-expanded T cells $(P<0.0001$; Figure $5 \mathrm{~B})$. We did not measure a statistically significant enhancement of the persistence of CAR-modified human $\mathrm{T}$ cells expanded under AKTi at a late (day 30) time point following infusion (Supplemental Figure 12). This finding might be attributable to the reduced half-life associated with CARs containing a CD28 costimulatory domain relative to other costimulatory domains, such as $4-1 \mathrm{BB}(15,35,36,40)$. Alternatively, given the variability in $\mathrm{T}$ cell engraftment observed between recipient mice, this study might have been underpowered to detect potentially small differences in persistence. Taken together, we conclude that anti-CD19 CAR-modified T cells expanded in the presence of AKTi treat established ALL superiorly compared with conventionally grown $\mathrm{T}$ cells. 


\section{Discussion}

Herein, we demonstrated that the eminently translatable strategy of adding a pharmacologic inhibitor specific for AKT-1/2 to a conventional $\mathrm{T}$ cell manufacturing process permitted efficient genetic engineering using both CARs and TCRs. Critically, this maneuver successfully uncoupled the processes of T cell expansion from phenotypic, functional, and metabolic maturation, resulting in a population of receptor-engineered T cells with enhanced in vivo antitumor efficacy. Mechanistically, the ability of AKTi to allow T cell expansion and transduction while preserving a minimally differentiated CD62L-expressing population was associated with conserved MAPK signaling and the intranuclear accumulation of FOXO1.

Genetic deletion of ERK2, a distal component of the MAPK pathway, was previously shown to impair $\mathrm{CD}^{+} \mathrm{T}$ cell proliferation and survival in mice (25). This finding mirrored the impaired in vitro proliferation of human T cells exposed to specific MAPK/extracellular signal-regulated kinase (MEK) inhibitors (26). Thus, preservation of MAPK signaling likely contributed to the preserved yield, enhanced cell viability, and uncompromised retroviral transduction efficiencies of human T cells we observed using AKTi. Despite the absence of a significant difference in cell expansion, we found that disruption of AKT signaling generates $\mathrm{T}$ cells with desirable phenotypic and functional attributes.

Our transcriptomic analyses of T cells transduced with an anti-CD19 CAR revealed that AKTi caused upregulation of genes associated with minimally differentiated T cells, including SELL. We noted that many of these genes were direct targets of FOXO1, a key transcriptional regulator of $\mathrm{T}$ cell memory formation (61) whose activity is negatively modulated by AKT. Using immunofluorescence microscopy, we discovered that AKTi caused a dose-dependent intranuclear localization of FOXO1. Because FOXO1 gene expression was unaltered by AKTi treatment, we hypothesized that a posttranslational mechanism might account for the subcellular accumulation of this protein. Expression of an AKT-resistant mutant of FOXO1 (FOXO1 ${ }^{\mathrm{AAA}}$ ) lacking the serine/threonine residues targeted by AKT directly phenocopied the influence of AKTi on CD62L, CD28, and TIM3 expression. Moreover, addition of AKTi to T cells expressing FOXO1 ${ }^{\text {AAA }}$ failed to further augment the frequency of $\mathrm{T}_{\mathrm{CM}}$ phenotype cells. Together, these findings indicate that preservation of FOXO1 activity was a major contributor of the AKT inhibitor effect. However, it is likely that AKTi also regulates transcriptional regulators other than FOXO1 in T cells. For example, the transcriptional repressor $\mathrm{BACH} 2$ was recently shown to also be a target of AKT phosphorylation and subsequent nuclear exclusion (75). Future studies will address whether BACH2 and FOXO3, the second most common FOXO family member expressed in lymphocytes (76), impact human $\mathrm{T}$ cell differentiation in an AKT-dependent manner.

Recent studies have established a causal link between cellular metabolism, T cell memory formation, and antitumor efficacy following ACT $(68,71,77)$. We found that AKTi suppressed the accumulation of lactate and attenuated the rate of extracellular acidification by human $\mathrm{T}$ cells, findings consistent with reduced aerobic glycolysis. The acquisition of a glycolytic metabolism during ex vivo cell expansion was previously shown to enhance $\mathrm{T}$ cell differentiation resulting in impaired in vivo antitumor efficacy. Although AKT plays a canonical role in regulating glucose metabolism across diverse cell types (70), a recent study in mice suggested that $\mathrm{AKT}$ is largely dispensable for short-term metabolic changes in $\mathrm{T}$ cells (27). However, 2 contemporaneous reports studying gain and loss of function of the PI3K/AKT pathway in human T cells came to a similar conclusion as ours $(72,78)$.

Gubser et al. demonstrated that pharmacologic inhibition of AKT isoforms 1/2 impaired an early glycolytic switch following human $\mathrm{T}$ cell activation (78). Lucas et al. showed that $\mathrm{T}$ cells from patients with gain-of-function mutations involving the PI3K p110 catalytic subunit had sustained AKT activation and enhanced glucose uptake, and they underwent spontaneous terminal differentiation (72). It is therefore possible that there are species-specific differences in the regulation of T cell glucose metabolism. Alternatively, sustained AKT signaling might regulate glucose metabolism through an indirect mechanism. For example, we found that gene-engineered $\mathrm{T}$ cells expanded for 10 days in AKTi had reduced expression of most of the glycolytic enzymes.

The adoptive transfer of $\mathrm{T}$ cells of defined composition offers the potential to have more consistent dose-response relationships between the number of $\mathrm{T}$ cells infused, antitumor efficacy, and potential toxicities (79). We and others have used $\mathrm{T}$ cell isolation techniques, such as streptamers $(58,80)$ or magnet bead isolation $(51,81)$, to create $\mathrm{T}$ cell populations with more uniform and desirable phenotypic and functional characteristics (5). In the present study, we used a bulk, unfractionated population of PBMCs to initiate our cultures, an approach used in most current $\mathrm{T}$ cell therapy trials. Despite interdonor variability in the 
distribution of $\mathrm{T}$ cell subsets, we found that cell expansion in AKTi gave rise to $\mathrm{T}$ cell populations with closely aligned gene expression and metabolic profiles. Thus, use of an AKT inhibitor offers a complementary strategy to physical cell isolation strategies to generate $\mathrm{T}$ cells with defined functional attributes across donors. We additionally demonstrated that AKTi is compatible with the isolation and anti-CD3/CD28 bead stimulation of $\mathrm{T}_{\mathrm{CM}}$ sorted cells. Thus, use of AKTi is compatible with the culture conditions currently used to generate therapeutic $\mathrm{T}$ cells in a wide range of academic centers and commercial groups.

We did not directly compare the in vivo antitumor activity of $\mathrm{T}$ cells retrovirally transduced with the FOXO1 ${ }^{\text {AAA }}$ construct relative to AKTi-treated cells. However, a recent study in mice by Luo et al. revealed that $\mathrm{T}$ cells expressing a murine version of FOXO1 ${ }^{\mathrm{AAA}}$ analogous to the human FOXO1 ${ }^{\mathrm{AAA}}$ used in our study led to an accumulation of $\mathrm{CD} 62 \mathrm{~L}^{+} \mathrm{T}$ cells in lymphoid organs and a corresponding reduction in TILs (82). These data suggest that dynamic control of FOXO1 activity in T cells is essential for permitting trafficking to peripheral tissues. It would therefore be important in future studies to compare the in vivo trafficking and antitumor activity of T cells grown in AKTi versus T cells engineered with either a constitutive or conditionally active version of FOXO1 $1^{\mathrm{AAA}}$

Our current findings build upon and significantly extend earlier studies demonstrating the utility of AKT inhibition in improving $\mathrm{T}$ cell therapies across a range of indications. For example, the efficiency of in vitro sensitization of allogenic minor histocompatibility-specific T cells (28) and the ex vivo expansion of melanoma TILs (29) were both improved following exposure to AKTi. More recently and consistent with our findings, Urak et al. reported that blockade of AKT activation prevented the terminal differentiation of CAR-modified $\mathrm{T}$ cells expanded to therapeutic doses (83).

In summary, we found that AKT inhibition permits efficient TCR and CAR genetic engineering and allows for the expansion of highly potent $\mathrm{CD} 62 \mathrm{~L}^{+} \mathrm{T}_{\mathrm{CM}}$ cells in a FOXO1-dependent manner. Despite starting with a bulk population, AKTi generates receptor-engineered T cells with defined genetic and metabolic properties and enhanced in vivo antitumor efficacy relative to conventionally produced $\mathrm{T}$ cells. These results are already influencing human $\mathrm{T}$ cell therapy clinical trial design. Based on our findings, the first human gene therapy clinical trial to incorporate AKTi in the T cell manufacturing process is now open to enrollment; this study has been registered at ClinicalTrials.gov (NCT03139370).

\section{Methods}

Culture media. Tumor cell lines were maintained in R10 medium, which consisted of RPMI 1640 medium (Lonza) supplemented with 10\% FBS (Hyclone), penicillin/streptomycin (100 U ml-1/100 $\mu \mathrm{G} \mathrm{ml}^{-1}$; Lonza), and 2 mM GlutaMAX (Invitrogen). T cells were maintained in T cell media, which consisted of AIMV (Invitrogen) supplemented with $5 \%$ human serum (Valley Biomedical), penicillin/streptomycin, nonessential amino acids (Gibco), GlutaMAX (Thermo Fisher Scientific), $0.055 \mathrm{mM}$ 2-mercaptoethanol (Gibco), and $50 \mathrm{CU} \mathrm{ml}^{-1}$ of rhIL-2 (Novartis). Where indicated, cells were cultured continuously with indicated concentrations of AKTi (Calbiochem) dissolved in DMSO (MilliporeSigma).

Cell lines. The following CD19+ cell lines were used as positive controls: NALM6 (DSMZ), NALM6GL (a previously described NALM6 line stably transduced with firefly luciferase) (58), and Toledo (ATCC). K562-CD19 (32) and 143B-CD19 (47) (a gift from C.L. Mackall, Stanford University, Stanford, California, USA) are both CD19- tumor cell lines stably transduced with CD19. The following CD19- cell lines were used as negative controls: K562 (ATCC) and CEM (ATCC).

Isolation, expansion, and retroviral transduction of human T cells with a clinical-grade CAR or TCR. Generation of anti-CD19 CAR-transduced human T cells was accomplished using a previously described manufacturing process identical to that currently used for prior and current human clinical trials (40). Briefly, unfractionated PBMC were obtained either by leukapheresis or venipuncture from healthy donors (HDs) or patients and prepared over Ficoll-Hypaque gradient (ICN Biomedicals Inc.). Cells were resuspended in IL-2 containing media (300 IU $\mathrm{ml}^{-1}$ ), and T cell proliferation was initiated using $50 \mathrm{ng} \mathrm{ml}^{-1}$ of anti-CD3 antibody (OKT3; OrthoBiotech). Alternatively, FACS-sorted $\mathrm{T}$ cell subsets were stimulated with anti-CD3/CD28 activator beads (Invitrogen) at 1:3 $\mathrm{T}$ cell/bead ratio. RetroNectin (Takara Bio Inc.) was applied to nontissue culture-coated 6-well plates using an established protocol (84), followed by addition of a previously described MSCV-based splice-gagvector (MSGV) retrovirus encoding either the FMC63-28z CAR (32) or a HLA-A*02 restricted NY-ESO-1 TCR (48). Two days after $\mathrm{T}$ cell stimulation, $2 \times 10^{6}$ stimulated $\mathrm{PBMC} /$ well were added to retrovirus-coated plates and subsequently cultured overnight at $37^{\circ} \mathrm{C}$. The next day, the cells were returned to culture in $\mathrm{T}$ cell media. Countbright beads were spiked-in for the flow cytometric quantification of relative and absolute cell numbers (Invitrogen). 
Antigen-specific functional assays. Specific IFN $\gamma$ release by anti-CD19 CAR-modified T cells was measured by ELISA assay (Pierce) from supernatants following overnight coculture at a 1:1 ratio with indicated $\mathrm{CD}_{1}{ }^{+}$and $\mathrm{CD} 19^{-}$target cell lines in IL-2-free/AKTi-free culture media. Intracellular cytokine staining (ICS) and tumor cell cytolysis was measured after a 6-hour coculture in a 1:2 ratio of anti-CD19 CAR-modified $\mathrm{T}$ cells to indicated tumor cell lines. Tumor cell cytotoxicity by receptor-engineered $\mathrm{T}$ cells and calculation of the tumor-elimination index was performed as described elsewhere (50). ICS was performed in the presence of $1 \mu \mathrm{l}$ of Golgi Plug (BD Pharmingen). Cells were subsequently washed, stained for viability and cell surface markers, permeabilized according to the manufacturer's instructions using a Cytofix/Cytoperm kit (BD Biosciences), and stained using fluorochrome conjugated antibodies against IFN $\gamma$ (clone RPA-T8; BioLegend) and IL-2 (clone MQ1-17H12; BD Biosciences). Evaluation of cytolysis by receptor-engineered $\mathrm{T}$ cells was performed as previously described (50) and calculated as the antigen-specific tumor elimination index.

Mouse xenograft ACT studies. Female NOD.Cg-Prkdcsid Il2 $\mathrm{rg}^{\text {tm } 1 \text { Wjil } / S z J}$ (The Jackson Laboratory) mice were used at $\geq 6$ weeks of age. Mice were inoculated by i.v. tail vein injection with $2 \times 10^{6}$ NALM6-GL cells. Three days later, mice were randomly assigned to receive either no T cells or $1 \times 10^{6}$ human antiCD19 CAR-transduced T cells expanded in the presence or absence of AKTi administered by i.v injection. No exogenous cytokine support was administered following adoptive transfer. Tumor burden was evaluated using bioluminescent imaging with a Xenogen Spectrum system and Living Image v3.2 software following i.p. administration of $150 \mathrm{mg} / \mathrm{kg}$ of D-luciferin (Caliper Life Sciences).

Antibodies and flow cytometry. Cells were stained with fluorochrome-conjugated antibodies against combinations of the following surface antigens: CD3 (SK7), CD4 (RPA-T4), CD8 (SK1), and CD62L (DREG-56) (all from BD Biosciences); CD28 (CD28.2, eBioscience); LAG-3 (17B4, Enzo); PD1 (MIH4, Invitrogen); and TIM-3 (344823, R\&D Systems). Fluorochrome conjugates against phoshpo-S6 S235/236 (D57.2.2E), phospho-Akt T308 (C31E5E), and phospho-Akt S473 (D9E) were obtained from Cell Signaling Technologies. Detection of the mouse anti-human CD19 scFv as a measure of transduction efficiency was detected as previously described (32). Briefly, Fc receptors were blocked with polyclonal goat IgG (Invitrogen), followed by incubation at $4^{\circ} \mathrm{C}$ for 25 minutes with biotin-labeled goat anti-mouse F(ab)2 antibody (Jackson ImmunoResearch). Cells were then blocked with polyclonal mouse IgG (Invitrogen) and then stained with PE-labeled streptavidin (BD Biosciences). Flow cytometric data was acquired using a either a BD FACSCanto II or LSRFortessa cytometer (BD Biosciences). FACS-sorting was performed on a FACSAria (BD Biosciences). FACS data was analyzed with FlowJo Version 9.8.5 software (TreeStar Inc.).

Metabolic profiling. ECAR was measured at $37^{\circ} \mathrm{C}$ using a Seahorse XFe96 Analyzer (Seahorse Bioscience) in XF media (nonbuffered RPMI 1640 containing $25 \mathrm{mM}$ glucose, $2 \mathrm{mM}$ L-glutamine, and 1 $\mathrm{mM}$ sodium pyruvate) under basal conditions as previously described (71). For FACS-based glucose uptake assays, T cells were incubated with $100 \mu \mathrm{M}$ 2-NBDG (Invitrogen) for 2 hours before measuring by FACS, also as previously described (71). T cells exposed to AKTi were maintained continuously in the presence of the inhibitor, including during incubation with 2-NBDG and during FACS analysis. Quantification of individual metabolites was accomplished using a combination of GC/MS and LC/ MS/MS platforms on CAR-modified T cells from 3 independent donors (Metabolon).

Microarray analysis. Gene expression levels were determined using Human Gene 1.0 ST arrays (Affymetrix) according to the manufacturer's protocols. Total RNA (300 ng) was used as starting material for cRNA amplification using the WT Expression kit (Invitrogen) according the manufacturer's protocol. cDNA was reverse transcribed, fragmented, labeled using the GeneChip WT Terminal Labeling kit (Affymetrix), and hybridized on the arrays for 18 hours according to the manufacturer's directions. Arrays were stained and washed in the Fluidics Station 400 (Affymetrix) and scanned (Affymetrix 7G). Arrays data were imported into Partek Genomic Suite using RMA normalization after background subtraction. The expression and sequencing data discussed in this manuscript are deposited in NCBI's GEO database and is accessible through the GEO series accession number GSE98078.

Confocal immunofluorescence imaging and analysis. Fluorescence image z-stacks were collected using a $63 \times$ plan-apochromat (numerical aperture) oil immersion objective lens on a Zeiss LSM 880 laser scanning confocal microscope (Carl Zeiss Microscopy Inc.). Tiled images were acquired with 5\% image overlap, an optical slice thickness of $0.9 \mathrm{um}, 0.07 \mathrm{um} \mathrm{x}$-y pixel size, and $0.44 \mathrm{um}$ z-step size. The z-stack image tiles were stitched using the Zen blue (v2.3) software. The stitched images were analyzed using 
the ImarisCell image analysis software (v. 8.2.1, Bitplane Inc.), with the Hoechst counterstain used to mask the nuclear volume and the phalloidin stain used to mask the cytoplasmic volume. The same image processing and analysis parameters were used for each of the images within a dataset. The mean intensity of the respective fluorescence channels was measured within the nucleus and cytoplasm, and the ratio of cytoplasmic to nuclear mean intensity was calculated for the individual cells. The multichannel fluorescence confocal image z-stacks were analyzed using the ImarisCell volume reconstruction module of the Imaris software. Briefly, within the ImarisCell module, the "cell with single nucleus" criterion was used to segment the nuclear volume compared with the cell cytoplasmic volume. The Hoechst fluorescence channel was used to segment the nucleus, whereas the phalloidin-Alexa Fluor 488 fluorescence channel was used to segment the cell cytoplasm. Segmentation was based primarily on intensity threshold for the respective channels above background. Background was calculated from the nonstained (no Hoechst and no phalloidin) negative control cells. From the 2 segmented compartments within the individual cells, the FOXO1 mean pixel intensity was measured in an automated fashion for both the nucleus and cytoplasm. The same parameters used for segmenting the cellular compartments were applied to all cells in the dataset, regardless of treatment assignment.

Statistics. Analysis of animal survival was assessed using a log-rank test. For all other experiments, data was compared using an unpaired 2-tailed Student's $t$ test corrected for multiple comparisons by a Bonferroni adjustment. In all cases, $P<0.05$ was considered significant. Statistics were calculated using the Prism 5 GraphPad software (GraphPad Software Inc.). For microarray data, 1 -way ANOVA was used to identify differentially expressed genes among the $4 \mathrm{~T}$ cell subtypes, with a significant cut off for the positive FDR $<0.01$. Differentially expressed probe sets were selected without specifying a fold change criterion.

Study approval. All human samples from anonymous NIH Blood Bank donors or cancer patients enrolled in clinical trials were approved by the NIH Clinical Center and NCI IRBs. Each patient signed an informed consent form and received a patient information form prior to participation. All mouse experiments were conducted under protocols approved by and in compliance with the NCI Bethesda Animal Care and Use Committee.

\section{Author contributions}

CAK, JGC, LG, SAF, and NPR designed and conceived of all experiments. SAF and MAB provided all retroviral constructs. MAB, TNY, AJL, SSC, DC, and SAF performed all tissue culture, retroviral transduction, and cytokine release experiments. JGC, AJL, MS, and DG performed and interpreted all functional metabolism studies. RLE performed all intracellular FACS analyses. LG, YJ, and JH performed all mouse xenograft experiments. RLE and SKV designed the FOXO1 retroviral construct. MJK and AJL designed and analyzed the immunofluorescence data. PJ and DFS generated and performed all transcriptomic analyses. PJ, JGC, and CAK interpreted all transcriptomic studies. CAK, SAF, and NPR provided supervision of the study. CAK and NPR wrote and edited the manuscript. All authors edited and commented on the final manuscript.

\section{Acknowledgments}

The authors thank Patricia Lopez for her excellent technical assistance with the Western blot analysis. This work was supported by startup funds from the MSKCC (CAK), the Parker Institute for Cancer Immunotherapy (CAK), the MSKCC Core grant P30 CA008748 (CAK), and the Intramural Research Program of the NCI, CCR of the US NIH (CAK, JGC, AJL, TNY, MS, RLE, SKV, DC, MB, DG, MK, PJ, DS, LG, SAF, NPR). The authors declare no competing financial interests.

Address correspondence to: Christopher A. Klebanoff, Memorial Sloan Kettering Cancer Center,

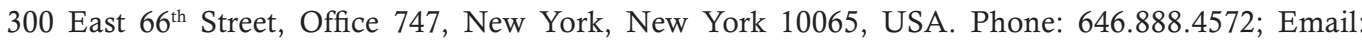
klebanoc@mskcc.org (C.A. Klebanoff). Or to: Nicholas P. Restifo, Center for Cancer Research, National Cancer Institute, 10 Center Drive, Building 10/CRC Room 3-5762, Bethesda, Maryland 20892, USA. Phone: 301.496.4904; Email: restifon@mail.nih.gov. 
1. Fry TJ, Mackall CL. T-cell adoptive immunotherapy for acute lymphoblastic leukemia. Hematology Am Soc Hematol Educ Program. 2013;2013:348-353.

2. Maus MV, June CH. Making Better Chimeric Antigen Receptors for Adoptive T-cell Therapy. Clin Cancer Res. 2016;22(8):1875-1884.

3. Klebanoff CA, Rosenberg SA, Restifo NP. Prospects for gene-engineered T cell immunotherapy for solid cancers. Nat Med. 2016;22(1):26-36

4. June CH, Blazar BR, Riley JL. Engineering lymphocyte subsets: tools, trials and tribulations. Nat Rev Immunol. 2009;9(10):704-716.

5. Klebanoff CA, Gattinoni L, Restifo NP. Sorting through subsets: which T-cell populations mediate highly effective adoptive immunotherapy? J Immunother. 2012;35(9):651-660.

6. Paulos CM, et al. Adoptive immunotherapy: good habits instilled at youth have long-term benefits. Immunol Res. 2008;42(1-3):182-196.

7. Klebanoff CA, et al. Determinants of successful CD8+ T-cell adoptive immunotherapy for large established tumors in mice. Clin Cancer Res. 2011;17(16):5343-5352.

8. Besser MJ, et al. Clinical responses in a phase II study using adoptive transfer of short-term cultured tumor infiltration lymphocytes in metastatic melanoma patients. Clin Cancer Res. 2010;16(9):2646-2655.

9. Radvanyi LG, et al. Specific lymphocyte subsets predict response to adoptive cell therapy using expanded autologous tumor-infiltrating lymphocytes in metastatic melanoma patients. Clin Cancer Res. 2012;18(24):6758-6770.

10. Goff SL, et al. Randomized, Prospective Evaluation Comparing Intensity of Lymphodepletion Before Adoptive Transfer of Tumor-Infiltrating Lymphocytes for Patients With Metastatic Melanoma. J Clin Oncol. 2016;34(20):2389-2397.

11. Robbins PF, et al. A pilot trial using lymphocytes genetically engineered with an NY-ESO-1-reactive T-cell receptor: long-term follow-up and correlates with response. Clin Cancer Res. 2015;21(5):1019-1027.

12. Rosenberg SA, et al. Durable complete responses in heavily pretreated patients with metastatic melanoma using T-cell transfer immunotherapy. Clin Cancer Res. 2011;17(13):4550-4557.

13. Louis CU, et al. Antitumor activity and long-term fate of chimeric antigen receptor-positive T cells in patients with neuroblastoma. Blood. 2011;118(23):6050-6056.

14. Fraietta JA, et al. Biomarkers of Response to Anti-CD19 Chimeric Antigen Receptor (CAR) T-Cell Therapy in Patients with Chronic Lymphocytic Leukemia. Blood. 2016;128(22):57.

15. Kochenderfer JN, et al. Lymphoma Remissions Caused by Anti-CD19 Chimeric Antigen Receptor T Cells Are Associated With High Serum Interleukin-15 Levels. J Clin Oncol. 2017;35(16):1803-1813.

16. Maus MV, Fraietta JA, Levine BL, Kalos M, Zhao Y, June CH. Adoptive immunotherapy for cancer or viruses. Annu Rev Immunol. 2014;32:189-225.

17. Gerlach C, et al. Heterogeneous differentiation patterns of individual CD8+ T cells. Science. 2013;340(6132):635-639.

18. Buchholz VR, et al. Disparate individual fates compose robust CD8+ T cell immunity. Science. 2013;340(6132):630-635

19. Kagoya Y, et al. Transient stimulation expands superior antitumor T cells for adoptive therapy. JCI Insight. 2017;2(2):e89580.

20. Bowers JS, et al. Th17 cells are refractory to senescence and retain robust antitumor activity after long-term ex vivo expansion. JCI Insight. 2017;2(5):e90772.

21. Klebanoff CA, et al. Memory T cell-driven differentiation of naive cells impairs adoptive immunotherapy. J Clin Invest. 2016;126(1):318-334.

22. Sommermeyer D, et al. Chimeric antigen receptor-modified T cells derived from defined CD8+ and CD4+ subsets confer superior antitumor reactivity in vivo. Leukemia. 2016;30(2):492-500.

23. Singh N, Perazzelli J, Grupp SA, Barrett DM. Early memory phenotypes drive T cell proliferation in patients with pediatric malignancies. Sci Transl Med. 2016;8(320):320ra3.

24. Gattinoni L, Klebanoff CA, Restifo NP. Paths to stemness: building the ultimate antitumour T cell. Nat Rev Cancer. 2012;12(10):671-684

25. D'Souza WN, Chang CF, Fischer AM, Li M, Hedrick SM. The Erk2 MAPK regulates CD8 T cell proliferation and survival. J Immunol. 2008;181(11):7617-7629.

26. Boni A, et al. Selective BRAFV600E inhibition enhances T-cell recognition of melanoma without affecting lymphocyte function. Cancer Res. 2010;70(13):5213-5219.

27. Macintyre AN, et al. Protein kinase B controls transcriptional programs that direct cytotoxic $\mathrm{T}$ cell fate but is dispensable for $\mathrm{T}$ cell metabolism. Immunity. 2011;34(2):224-236.

28. van der Waart AB, et al. Inhibition of Akt signaling promotes the generation of superior tumor-reactive $\mathrm{T}$ cells for adoptive immunotherapy. Blood. 2014;124(23):3490-3500.

29. Crompton JG, et al. Akt inhibition enhances expansion of potent tumor-specific lymphocytes with memory cell characteristics. Cancer Res. 2015;75(2):296-305.

30. Lewis PF, Emerman M. Passage through mitosis is required for oncoretroviruses but not for the human immunodeficiency virus. J Virol. 1994;68(1):510-516.

31. Calleja V, Laguerre M, Parker PJ, Larijani B. Role of a novel PH-kinase domain interface in PKB/Akt regulation: structural mechanism for allosteric inhibition. PLoS Biol. 2009;7(1):e17.

32. Kochenderfer JN, et al. Construction and preclinical evaluation of an anti-CD19 chimeric antigen receptor. J Immunother. 2009;32(7):689-702.

33. Locke FL, et al. Phase 1 Results of ZUMA-1: A Multicenter Study of KTE-C19 Anti-CD19 CAR T Cell Therapy in Refractory Aggressive Lymphoma. Mol Ther. 2017;25(1):285-295.

34. Maude SL, et al. Chimeric antigen receptor T cells for sustained remissions in leukemia. N Engl J Med. 2014;371(16):1507-1517.

35. Davila ML, et al. Efficacy and toxicity management of 19-28z CAR T cell therapy in B cell acute lymphoblastic leukemia. Sci Transl Med. 2014;6(224):224ra25

36. Lee DW, et al. T cells expressing CD19 chimeric antigen receptors for acute lymphoblastic leukaemia in children and young adults: a phase 1 dose-escalation trial. Lancet. 2015;385(9967):517-528. 
37. Kebriaei P, et al. Phase I trials using Sleeping Beauty to generate CD19-specific CAR T cells. J Clin Invest. 2016;126(9):3363-3376.

38. Porter DL, et al. Chimeric antigen receptor T cells persist and induce sustained remissions in relapsed refractory chronic lymphocytic leukemia. Sci Transl Med. 2015;7(303):303ra139.

39. Garfall AL, et al. Chimeric Antigen Receptor T Cells against CD19 for Multiple Myeloma. N Engl J Med. 2015;373(11):1040-1047.

40. Kochenderfer JN, et al. Chemotherapy-refractory diffuse large B-cell lymphoma and indolent B-cell malignancies can be effectively treated with autologous T cells expressing an anti-CD19 chimeric antigen receptor. J Clin Oncol. 2015;33(6):540-549.

41. Brudno JN, et al. Allogeneic T Cells That Express an Anti-CD19 Chimeric Antigen Receptor Induce Remissions of B-Cell Malignancies That Progress After Allogeneic Hematopoietic Stem-Cell Transplantation Without Causing Graft-Versus-Host Disease. J Clin Oncol. 2016;34(10):1112-1121.

42. DeSilva DR, et al. Inhibition of mitogen-activated protein kinase kinase blocks $\mathrm{T}$ cell proliferation but does not induce or prevent anergy. J Immunol. 1998;160(9):4175-4181.

43. Finlay DK, et al. PDK1 regulation of mTOR and hypoxia-inducible factor 1 integrate metabolism and migration of CD8+ T cells. J Exp Med. 2012;209(13):2441-2453.

44. Klebanoff CA, et al. Central memory self/tumor-reactive CD8+ T cells confer superior antitumor immunity compared with effector memory T cells. Proc Natl Acad Sci USA. 2005;102(27):9571-9576.

45. Abu Eid R, et al. Akt1 and -2 inhibition diminishes terminal differentiation and enhances central memory CD8(+) T-cell proliferation and survival. Oncoimmunology. 2015;4(5):e1005448.

46. Frigault MJ, et al. Identification of chimeric antigen receptors that mediate constitutive or inducible proliferation of $\mathrm{T}$ cells. Cancer Immunol Res. 2015;3(4):356-367.

47. Long AH, et al. 4-1BB costimulation ameliorates $\mathrm{T}$ cell exhaustion induced by tonic signaling of chimeric antigen receptors Nat Med. 2015;21(6):581-590.

48. Robbins PF, et al. Single and dual amino acid substitutions in TCR CDRs can enhance antigen-specific T cell functions. J Immunol. 2008;180(9):6116-6131.

49. Rapoport AP, et al. NY-ESO-1-specific TCR-engineered T cells mediate sustained antigen-specific antitumor effects in myeloma. Nat Med. 2015;21(8):914-921.

50. Mastaglio S, et al. NY-ESO-1 TCR single edited stem and central memory T cells to treat multiple myeloma without graftversus-host disease. Blood. 2017;130(5):606-618.

51. Turtle CJ, et al. CD19 CAR-T cells of defined CD4+:CD8+ composition in adult B cell ALL patients. J Clin Invest. 2016;126(6):2123-2138.

52. Graef $\mathrm{P}$, et al. Serial transfer of single-cell-derived immunocompetence reveals stemness of CD8(+) central memory T cells. Immunity. 2014;41(1):116-126.

53. Powell DJ, Dudley ME, Robbins PF, Rosenberg SA. Transition of late-stage effector T cells to CD27+ CD28+ tumor-reactive effector memory T cells in humans after adoptive cell transfer therapy. Blood. 2005;105(1):241-250.

54. Chandran SS, Paria BC, Srivastava AK, Rothermel LD, Stephens DJ, Kammula US. Tumor-Specific Effector CD8+ T Cells That Can Establish Immunological Memory in Humans after Adoptive Transfer Are Marked by Expression of IL7 Receptor and c-myc. Cancer Res. 2015;75(16):3216-3226

55. Cruz AC, et al. Fas/CD95 prevents autoimmunity independently of lipid raft localization and efficient apoptosis induction. Nat Commun. 2016;7:13895.

56. Kägi D, et al. Fas and perforin pathways as major mechanisms of T cell-mediated cytotoxicity. Science. 1994;265(5171):528-530.

57. Barrett DM, et al. Relation of clinical culture method to T-cell memory status and efficacy in xenograft models of adoptive immunotherapy. Cytotherapy. 2014;16(5):619-630.

58. Sabatino M, et al. Generation of clinical-grade CD19-specific CAR-modified CD8+ memory stem cells for the treatment of human B-cell malignancies. Blood. 2016;128(4):519-528.

59. Gattinoni L, et al. Acquisition of full effector function in vitro paradoxically impairs the in vivo antitumor efficacy of adoptively transferred CD8+ T cells. J Clin Invest. 2005;115(6):1616-1626.

60. Kerdiles YM, et al. Foxo1 links homing and survival of naive T cells by regulating L-selectin, CCR7 and interleukin 7 receptor. Nat Immunol. 2009;10(2):176-184

61. Hess Michelini R, Doedens AL, Goldrath AW, Hedrick SM. Differentiation of CD8 memory T cells depends on Foxo1. J Exp Med. 2013;210(6):1189-1200.

62. Rao RR, Li Q, Gubbels Bupp MR, Shrikant PA. Transcription factor Foxo1 represses T-bet-mediated effector functions and promotes memory CD8(+) T cell differentiation. Immunity. 2012;36(3):374-387.

63. Kim MV, Ouyang W, Liao W, Zhang MQ, Li MO. The transcription factor Foxol controls central-memory CD8+ T cell responses to infection. Immunity. 2013;39(2):286-297.

64. Rena G, Guo S, Cichy SC, Unterman TG, Cohen P. Phosphorylation of the transcription factor forkhead family member FKHR by protein kinase B. J Biol Chem. 1999;274(24):17179-17183.

65. Greer EL, Brunet A. FOXO transcription factors at the interface between longevity and tumor suppression. Oncogene. 2005;24(50):7410-7425

66. Zhang X, et al. Phosphorylation of serine 256 suppresses transactivation by FKHR (FOXO1) by multiple mechanisms. Direct and indirect effects on nuclear/cytoplasmic shuttling and DNA binding. J Biol Chem. 2002;277(47):45276-45284.

67. Fabre S, et al. FOXO1 regulates L-Selectin and a network of human T cell homing molecules downstream of phosphatidylinositol 3-kinase. J Immunol. 2008;181(5):2980-2989.

68. Kawalekar OU, et al. Distinct Signaling of Coreceptors Regulates Specific Metabolism Pathways and Impacts Memory Development in CAR T Cells. Immunity. 2016;44(3):712.

69. Milone MC, et al. Chimeric receptors containing CD137 signal transduction domains mediate enhanced survival of $\mathrm{T}$ cells and increased antileukemic efficacy in vivo. Mol Ther. 2009;17(8):1453-1464.

70. Plas DR, Thompson CB. Akt-dependent transformation: there is more to growth than just surviving. Oncogene. 
2005;24(50):7435-7442.

71. Sukumar M, et al. Inhibiting glycolytic metabolism enhances CD8+ T cell memory and antitumor function. $J$ Clin Invest. 2013;123(10):4479-4488.

72. Lucas CL, et al. Dominant-activating germline mutations in the gene encoding the PI(3)K catalytic subunit p110 $\delta$ result in $\mathrm{T}$ cell senescence and human immunodeficiency. Nat Immunol. 2014;15(1):88-97.

73. Wieman HL, Wofford JA, Rathmell JC. Cytokine stimulation promotes glucose uptake via phosphatidylinositol-3 kinase/Akt regulation of Glut1 activity and trafficking. Mol Biol Cell. 2007;18(4):1437-1446.

74. Miyamoto S, Murphy AN, Brown JH. Akt mediates mitochondrial protection in cardiomyocytes through phosphorylation of mitochondrial hexokinase-II. Cell Death Differ. 2008;15(3):521-529.

75. Roychoudhuri R, et al. BACH2 regulates CD8(+) T cell differentiation by controlling access of AP-1 factors to enhancers Nat Immunol. 2016;17(7):851-860.

76. Hedrick SM, Hess Michelini R, Doedens AL, Goldrath AW, Stone EL. FOXO transcription factors throughout T cell biology. Nat Rev Immunol. 2012;12(9):649-661.

77. Sukumar M, et al. Mitochondrial Membrane Potential Identifies Cells with Enhanced Stemness for Cellular Therapy. Cell Metab. 2016;23(1):63-76

78. Gubser PM, et al. Rapid effector function of memory CD8+ T cells requires an immediate-early glycolytic switch. Nat Immunol. 2013;14(10):1064-1072.

79. Riddell SR, et al. Adoptive therapy with chimeric antigen receptor-modified $\mathrm{T}$ cells of defined subset composition. Cancer J. 2014;20(2):141-144.

80. Stemberger $\mathrm{C}$, et al. Novel serial positive enrichment technology enables clinical multiparameter cell sorting. PLoS ONE. 2012;7(4):e35798

81. Terakura S, Yamamoto TN, Gardner RA, Turtle CJ, Jensen MC, Riddell SR. Generation of CD19-chimeric antigen receptor modified CD8+ T cells derived from virus-specific central memory T cells. Blood. 2012;119(1):72-82.

82. Luo CT, Liao W, Dadi S, Toure A, Li MO. Graded Foxo1 activity in Treg cells differentiates tumour immunity from spontaneous autoimmunity. Nature. 2016;529(7587):532-536.

83. Urak R, et al. Ex vivo Akt inhibition promotes the generation of potent CD19CAR T cells for adoptive immunotherapy J Immunother Cancer. 2017;5:26.

84. Johnson LA, et al. Gene therapy with human and mouse T-cell receptors mediates cancer regression and targets normal tissues expressing cognate antigen. Blood. 2009;114(3):535-546. 